\title{
Screening and characterization of four $\beta$-lactam antibiotic degradation strains PG102
}

\author{
Zinuo Wang, Yanju Li * \\ School of life science, Beijing Institute of Technology, Beijing 100000, China \\ *Corresponding author e-mail: LYJ_bit721@163.com
}

Keywords: PG102, $\beta$-lactam, antibiotic.

\begin{abstract}
In this study, a strain PG102 capable of growing with $\beta$-lactam antibiotics as the sole carbon and nitrogen source was isolated from fresh pig manure. Amoxicillin, cefuroxime sodium, and penicillin potassium 3 were cultured within 3 days. Several $\beta$-lactam antibiotics were completely degraded and identified as Achromobacter anxifer. Addition of glucose carbon source and $\mathrm{NH} 4 \mathrm{Cl}$ nitrogen source can accelerate its degradation. Within 12 hours, amoxicillin, cefuroxime sodium, and penicillin potassium three kinds of mixed antibiotics with a concentration of $100 \mathrm{mg} / \mathrm{L}$ can be completely degraded. The bacteria can grow at $55^{\circ} \mathrm{C}$, have good heat resistance, can be completely degraded at $\mathrm{pH} 5-9$,and the minimum degradation time is $12 \mathrm{~h}$ at $\mathrm{pH}$ 8-9. This provides a reference for the antibiotic environmental pollution treatment of livestock and poultry waste.
\end{abstract}

\section{Introduction}

In the process of livestock and poultry breeding, many antibiotics are added, among which the wildlife-lactam antibiotics are widely used in animal husbandry [1,2,]. $\beta$-lactamantibiotics cannot be completely absorbed by the body, but most of them are excretedby feces in the form of protoplasm or metabolites, and eventually adsorb and accumulate in the soil along with manure for agricultural use, causing damage to the ecological environment and posing challenges to the sustainable development of animal husbandry [3,4].Therefore, in order to solve the problem of environmental pollution of antibiotics, degradation of da-lactam antibiotics is urgently needed.

The degradation of -lactam antibiotics can be divided into physicochemical method and biological method [5].Among them, biodegradation has become an important means of self-purification of antibiotics in soil due to its advantages of high sustainability, thorough degradation, strong specificity, low cost and no secondary pollution [6,7].Biodegradation plays an important role in antibiotic degradation of livestock and poultry waste [8].How to obtain a large number of strains with strong environmental adaptability and high degradation efficiency, and make appropriate agents for use is an urgent problem to be solved.For this reason, the isolation of efficient degradation strains has attracted extensive attention.In 2014, Erickson et al. [9] isolated a strain capable of efficiently degrading ceftiofur from cow dung. It was identified that the strain was Bacillus cereus.In 2015, Lin et al. [10] screened two cephalexin-degradable strains from activated sludge and named them Pseudomonas sp. EC21 and Pseudomonas sp. EC22.Although many scientists have done related work in this area before, some of the selected strains cannot completely degrade antibiotics, or it takes a long time for complete degradation. To this end, this study attempts to screen out strains with high degradation ability from pig manure, and to study their biological and degradation characteristics in order to provide a scientific basis for the environmental management of antibiotics for livestock waste. 


\section{Materials and methods}

\subsection{Experimental Materials}

(1) Livestock and poultry waste: collected from an agricultural and animal husbandry science and technology company in Inner Mongolia

(2) Antibiotics: Amoxicillin (The following abbreviations are AMX), purchased from North China Pharmaceutical Factory, purity 98\%; Cefuroxime sodium (The following abbreviations are Cefur) , purchased from North China Pharmaceutical Factory, purity 98\%; Penicillin potassium (The following abbreviations are PG), purchased from North China Pharmaceutical Factory, purity 98\%; Ceftiofur Sodium (The following abbreviations are Cefti), purchased from Yuanye Biological Company, purity $98 \%$.

Medium: Inorganic salt medium: KH2PO4 0.50 g, $\mathrm{K}_{2} \mathrm{HPO}_{4} 0.50 \mathrm{~g}, \mathrm{NaCl} 0.20$ g, $\mathrm{NH}_{4} \mathrm{NO}_{3} 2.00$ g, $\mathrm{MgSO}_{4} \cdot 7 \mathrm{H}_{2} \mathrm{O} 0.20 \mathrm{~g}, \mathrm{CaCl}_{2} 0.10 \mathrm{~g}, \mathrm{FeSO}_{4} \cdot 7 \mathrm{H}_{2} \mathrm{O} 0.01 \mathrm{~g}, \mathrm{MnSO}_{4} 0.01 \mathrm{~g}, \mathrm{H}_{2} \mathrm{O} 1 \mathrm{~L}$

Beef extract peptone medium: Beef extract 5.00 g, peptone $10.00 \mathrm{~g}, \mathrm{NaCl} 5.00 \mathrm{~g}, \mathrm{H}_{2} \mathrm{O} 1 \mathrm{~L}$

\subsection{Experimental method}

2.2.1 Screening and identification of $\beta$-lactam antibiotic degradation strains

(1) Strain screening. 25\% fresh pig manure was inoculated in a liquid medium of inorganic salts with different -lactam antibiotics as the only carbon source.The domestication concentration was $100 \mathrm{mg} / \mathrm{L}$.At $30^{\circ} \mathrm{C}$ and $180 \mathrm{r}$, the concentration gradient increased every 48 hours.200ul enrichment solution of bacterial species was taken, coated and diluted by coating method, and then cultured at $30^{\circ} \mathrm{C}$ for $1-3 \mathrm{~d}$. The bacteriostatic circle method was used for screening.Doxi-lactam antibiotics including 100mg/L4 different amoxicillin, cefuroxime sodium, cefuroxime sodium and other doxi-lactam antibiotics were respectively inoculated with a dose of $15 \%$, and the bacteriostatic zone at day 0, 2, 4 and 6 was determined, with a degradation rate of more than 50\%.The antibiotic degradation rate A\% was calculated according to formula 1.1.(the following experimental method of bacteriostasis circle is the same as the calculation formula.)

$$
\mathrm{A} \%=\left(\mathrm{C}_{\mathrm{CKn}}-\mathrm{C}_{\mathrm{n}}\right) / \mathrm{C}_{\mathrm{CKn}} * 100 \%
$$

In the formula, $\mathrm{C}_{\mathrm{CKn}}$ represents the average diameter of the inhibition zone of the control group on the nth day, and $\mathrm{C}_{n}$ represents the average diameter of the inhibition zone of the experimental group on the nth day. By comparing the strain inhibition zones, several strains with the highest degradation rate were obtained for further investigation.

Strain identification. In this experiment, the total DNA of the strain was extracted using a kit method. A $2 \mu \mathrm{L}$ gDNA sample was taken and detected by $1.0 \%$ agarose gel electrophoresis, while 3 $\mu \mathrm{L}$ of DL2000Marker was used as a control. The entire sequence of the strain was amplified using universal primers. The primers were synthesized by Biotech Bio (Shanghai) Co., Ltd.

\subsubsection{Study on Growth Characteristics of Strains}

(1) Strain growth curve. The strain was inoculated in beef extract liquid medium and cultured at $30^{\circ} \mathrm{C}, 160 \mathrm{r} / \mathrm{min}$. From $0 \mathrm{~h}$, the ultraviolet spectrophotometry method was used to sample and measure the absorbance of OD600nm of the strain, and the strain growth curve was drawn according to the turbidity.

(2) Determination of strain heat resistance. The higher the heat resistance of the strains The more favorable it is for practical applications, it is necessary to investigate the heat resistance of the strains. Using the dilution coating plate method, take $1 \mathrm{ml}$ of bacterial solution into $9 \mathrm{ml}$ of sterile physiological saline, that is, dilute to $10-1$, and then dilute to $10-6$ dilution concentration in sequence. Take 200ul of 10-4, 10-5, and 10-6 solutions, coat them on a solid plate of beef paste, and incubate at $35^{\circ} \mathrm{C}, 45^{\circ} \mathrm{C}$, and $55^{\circ} \mathrm{C}$ for $1-2$ days, and count the number of colonies growing. Colony calculation formula: CFU per ml of bacterial solution average

Value of plate CFU / dilution factor / 0.2 


\subsubsection{Study on Degradation Characteristics of Strains}

(1) Degradation experiment of strain PG102 using antibiotics as the sole carbon and nitrogen source. Take $3 \mathrm{ml}$ of the bacterial solution, centrifuge at $4000 \mathrm{r}$, 4min, discard the supernatant, take the bacterial cells to the inorganic salt liquid medium, and then add $100 \mathrm{mg} / \mathrm{L} \beta$ - Lactam antibiotics (cefuroxime sodium: ceftiofur sodium: penicillin potassium: amoxicillin $=1: 1: 1$ : 1 each of the four antibiotics are mixed at $25 \mathrm{mg} / \mathrm{L}$ ), and antibiotics are the only C and N sources. Samples were taken every $12 \mathrm{~h}$ from $0 \mathrm{~h}$ to determine the size of the inhibition zone, and the degradation rate was compared. The dynamic curves of antibiotic degradation were drawn and calculated by the first-order reaction degradation kinetics formula. The degradation kinetics formula, half-life and $\mathrm{k}$ formula are shown in 1.2 and 1.3.

$$
\begin{aligned}
\mathrm{C}_{\mathrm{t}} & =\mathrm{C}_{0} \mathrm{e}^{-\mathrm{kt}} \\
\mathrm{t}_{50} & =\ln 2 / \mathrm{k}
\end{aligned}
$$

In the formula, $\mathrm{k}$ is the first order kinetic degradation rate, $\mathrm{Ct}$ is the content of antibiotics at time $\mathrm{t}$, C0 is the content of antibiotics at time 0 , and $\mathrm{t} 50$ is the time required for the degradation of antibiotics at $50 \%$.

(2) Degradation experiment of strain PG102 using glucose as a co-metabolized carbon source. The carbon source was selected to be $500 \mathrm{mg} / \mathrm{L}$ glucose. Glucose was added to the inorganic salt liquid medium.The degradation kinetics formula, half-life and $\mathrm{k}$ formula are shown in 1.1 and 1.2.

Degradation experiment of strain PG102 using $\mathrm{NH} 4 \mathrm{Cl}$ as a co-metabolizing nitrogen source. The carbon source was selected as $500 \mathrm{mg} / \mathrm{L}$ glucose and the nitrogen source was $\mathrm{NH} 4 \mathrm{Cl}$. After consulting the literature, it was found that when the carbon-nitrogen ratio was set to 10: 1, it was favorable for strain degradation. 10: 1 carbon-nitrogen ratio was added NH4Cl. The method of adding antibiotics and inhibition zone is the same as above. The degradation kinetics formula, half-life and $\mathrm{k}$ formula are shown in 1.1 and 1.2.

Suitable $\mathrm{pH}$ test for strain degradation. the degradation conditions were further optimized on the basis of the co-metabolic carbon and nitrogen sources. The carbon and nitrogen sources were consistent with the above experimental Settings, namely carbon (glucose) $500 \mathrm{mg} / \mathrm{L}$, nitrogen $(\mathrm{NH} 4 \mathrm{Cl}$ ), and the ratio of carbon and nitrogen sources was 10:1.and the $\mathrm{pH}$ was set to 5, 6, 7, 8, 9.

\section{Results}

\subsection{Strain screening and identification of $\beta$-lactam antibiotic degradation strains}

(1) Strain screening. Four kinds of lactam antibiotics were selected: cefuroxime sodium, cefutiroxime sodium, penipotassium and amoxicillin. The concentration was set at $100 \mathrm{mg} / \mathrm{L}$. The degradation rate was obtained by measuring the diameter of the inhibition circle using the bacteriostatic circle method.PG102, which had high degradation rate of all four antibiotics, was selected for the next experiment.

It can be seen from Fig. 1 that the strain PG102 can degrade amoxicillin, cefuroxime sodium, ceftiofur sodium, and penicillin potassium 4 kinds of $\beta$-lactam antibiotics, which has a wide range of degradation, a wide degradation spectrum, and high degradation efficiency. When penicillin potassium was used as the sole carbon and nitrogen source, the degradation rate was $100 \%$ on the third day; when amoxicillin was used as the sole carbon and nitrogen source, the degradation rate was $100 \%$ on the 4 th day; cefuroxime sodium was used as the sole carbon. At the time of source, the degradation rate of $2 \mathrm{~d}$ reached $100 \%$; when ceftiofur sodium was used as the sole carbon source, although it did not reach complete degradation, the degradation rate of $5 \mathrm{~d}$ also reached $50.54 \%$. 


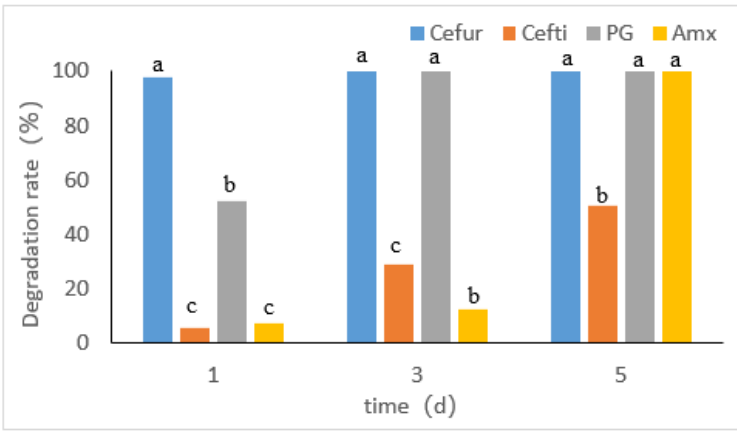

Figure 1. Degradation of 4 kinds of $\beta$-lactam antibiotics by strain PG102

(2)Strain identification. The colony morphology of strain PG102 are shown in Fig. 2a.16S rRNA gene identification was performed on this strain. Theresults showed that the strain PG102 had the most recent homology with Achromobacter anxifer. The EZbiocloud results showed 100\% homology. Therefore, the strain PG-10-2 was determined Achromobacter anxifer. Phylogenetic tree of PG102 is shown in Fig. $2 b$.

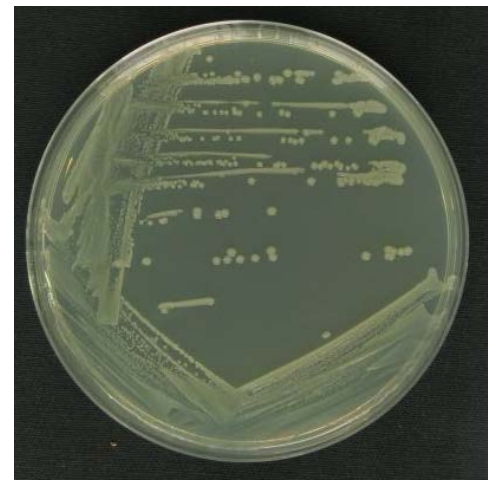

(a)

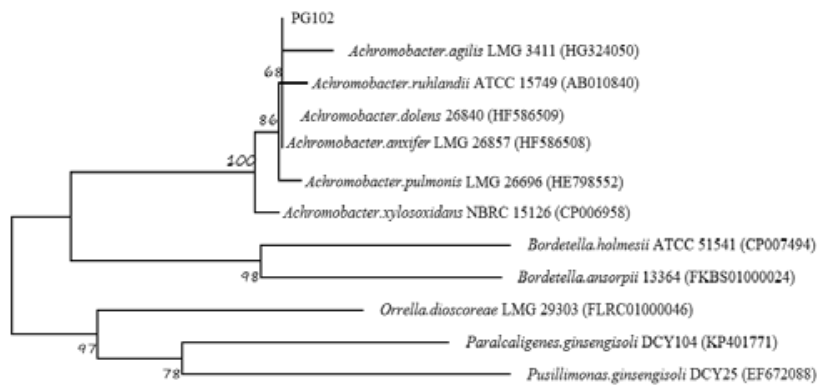

(b)

Figure 2. Strain morphology and phylogenetic tree of PG102

\subsection{Strain growth curve}

Under beef paste culture conditions, the growth retardation period of strain PG102 is 0-4h, the exponential period is $4-24 \mathrm{~h}$, and the plateau period is $24 \mathrm{~h}$. The highest OD value is 4.96 . The biomass is larger and the growth rate is faster.

\subsection{Determination of strain heat resistance}

The total CFU of $1 \mathrm{ml}$ of bacterial strain of strain $\mathrm{PG} 102$ at $35^{\circ} \mathrm{C}$ is $6.70 \times 10^{8} \mathrm{CFU} / \mathrm{ml}$; the total $\mathrm{CFU}$ of strain $\mathrm{Am} 101$ at $45^{\circ} \mathrm{C}$ is $5.10 \times 10^{8} \mathrm{CFU} / \mathrm{ml} ; 55^{\circ} \mathrm{C}$ The total $\mathrm{CFU}$ of the lower strain Am101 was $4.53 \times 10^{8} \mathrm{CFU} / \mathrm{ml}$. However, if $35^{\circ} \mathrm{C}>45^{\circ} \mathrm{C}>55^{\circ} \mathrm{C}$, the total number of colonies at $35^{\circ} \mathrm{C}$ is 1.3 times the total number of colonies at $45^{\circ} \mathrm{C}$; the total number of colonies at $45^{\circ} \mathrm{C}$ is 112 times the total number of colonies at $55^{\circ} \mathrm{C}$. This shows that $\mathrm{PG} 102$ can grow at $35^{\circ} \mathrm{C}, 45^{\circ} \mathrm{C}$, and $55^{\circ} \mathrm{C}$, but $35^{\circ} \mathrm{C}$ is a suitable temperature for the growth of the strain, and $55^{\circ} \mathrm{C}$ is a high temperature condition that the strain can tolerate, but not its suitable growth temperature.

\subsection{Effects of co-metabolism of external carbon and nitrogen sources on degradation of strain PG102}

Strain PG102 has higher degradation effect when $\beta$-lactam antibiotics are used as the sole carbon source, but there are certain problems. One is that ceftiofur sodium is not the only $\mathrm{C}$ and $\mathrm{N}$ source within 5 days. Degradation; Second, other ceffurin sodium, amoxicillin, and penicillin potassium were the only $\mathrm{C}$ and $\mathrm{N}$ sources that did not reach complete degradation within $1 \mathrm{~d}$ or less. Therefore, in order to further improve the degradation efficiency of the strain, Co-metabolism experiments 
with additional carbon source (glucose) and additional carbon and nitrogen sources (carbon source: glucose; nitrogen source: $\mathrm{NH} 4 \mathrm{Cl}$; carbon-nitrogen ratio 10: 1) were conducted to compare the degradation effect.

It can be obtained from Fig. 3a that the strain PG102 is treated with $\beta$-lactam antibiotics (cefuroxime sodium: ceftiofur sm: penicillin potassium: amoxicillin $=1: 1: 1$ : 1 , cefuroxime sodium + ceftiofur Sodium + penicillin potassium + amoxicillin $=100 \mathrm{mg} / \mathrm{L}$ ) is the only source of C and N, complete degradation can be achieved in 36 hours; but when co-metabolized with $500 \mathrm{mg} / \mathrm{L}$ glucose as carbon source, complete degradation is achieved in 24 hours, compared with The degradation of $\beta$-lactam antibiotics as the only $\mathrm{C}$ and $\mathrm{N}$ sources was shortened by 12 hours; when glucose was added as a co-metabolic carbon source, and $\mathrm{NH} 4 \mathrm{Cl}$ was added as a co-metabolic nitrogen source, complete degradation was achieved within $12 \mathrm{~h}$, compared with only glucose as a co-metabolic source. The metabolic carbon source was shortened by 12 hours, and it was shortened by 24 hours compared with antibiotics as the only $\mathrm{C}$ and $\mathrm{N}$ sources.It can be obtained from Fig. $3 \mathrm{~b}$ that the strain PG102 can grow in all three conditions, and the biomass has increased. Compared with the OD value of day 0 , the combined carbon source metabolism increased by 0.3 , and the combined carbon and nitrogen source metabolism Increased by 0.15 , and the sole carbon and nitrogen source co-metabolism increased by 0.074 . The $\mathrm{pH}$ is basically maintained between 6.10-6.60.

The table 1 shows that strain PG102 antibiotics as the sole carbon and nitrogen source of degradation speed slower than other two, long half-life, $34.88 \mathrm{~h}$, and the strain PG102 plus metabolism of carbon and nitrogen source were cases, half-life is the shortest, $12.876 \mathrm{~h}$, is known best price effect, the second is in plus carbon metabolism, also had a shorter half-life,14.89h.

a

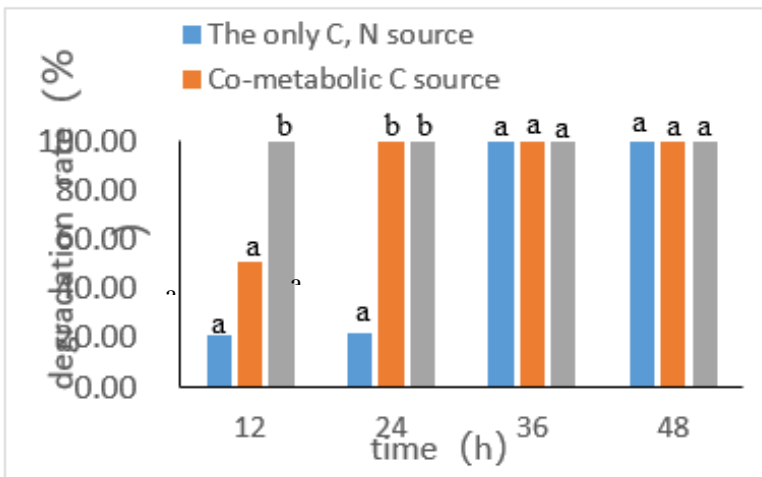

b

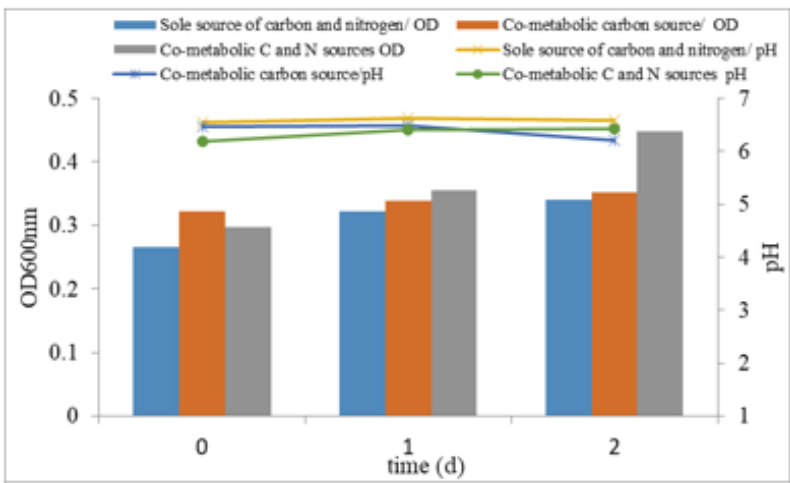

Figure 3. Degradation of $\beta$-lactam antibiotics by strain PG102 under three C、N sources a: Histogram of degradation rate b:OD600nm value and $\mathrm{pH}$ value chart

Table1. Kinetic parameters of degradation of $\beta$-lactam antibiotics by strain PG102 under three carbon and nitrogen source conditions

\begin{tabular}{|c|c|c|c|c|}
\hline Carbon and nitrogen source conditions & $\begin{array}{c}\text { Degradation kinetics } \\
\text { equation }\end{array}$ & $\begin{array}{c}\mathrm{C}_{0} \\
/ 10^{-3} \\
\end{array}$ & $\mathrm{k}$ & $\mathrm{t}_{50} / \mathrm{h}$ \\
\hline $\begin{array}{l}\beta \text {-lactam antibiotics are the only source of } \\
\text { carbon and nitrogen }\end{array}$ & $C_{t}=0.05 e^{-0.02 t}$ & 0.05 & 0.020 & 34.88 \\
\hline Cometabolic carbon source (glucose) & $C_{t}=0.05 e^{-0.047 t}$ & 0.05 & 0.047 & 14.89 \\
\hline $\begin{array}{l}\text { Co- metabolized carbon and nitrogen sources } \\
\text { (glucos, } \mathrm{NH}_{4} \mathrm{Cl} \text { ) }\end{array}$ & $C_{t}=0.05 e^{-0.054 t}$ & 0.05 & 0.054 & 12.876 \\
\hline
\end{tabular}




\subsection{Effect of initial pH on degradation of strain PG102}

The degradation rates and kinetics equations of bacterial strains at different $\mathrm{pH}$ values are shown in figure 3 and table 2.It can be seen from Fig 4a that strain PG102 can completely degrade $\beta$-lactam antibiotics in the range of $\mathrm{pH} 5-9$. When the $\mathrm{pH}$ is 8,9 , the degradation rate reaches $100 \%$ at $12 \mathrm{~h}$, indicating that $\mathrm{pH} 8-9$ is the optimal $\mathrm{pH}$ for the degradation of $\beta$-lactam antibiotics by strain PG102. It can be seen from Fig. $4 \mathrm{~b}$ that as the degradation time increases, the biomass of the strain PG102 increases, which indicates that the strain can degrade antibiotics under three conditions: the sole carbon and nitrogen source, the co-metabolic carbon source, and the co-metabolic carbon and nitrogen source. In order to obtain growth; the $\mathrm{pH}$ of the inorganic salt liquid medium at $1 \mathrm{~d}$ and $2 \mathrm{~d}$ after the addition of the strain PG102 generally decreased, which may be because the strain PG102 itself was acidic, or the degradation products produced by the strain degrading antibiotics were acidic.

At different $\mathrm{pH}$ values, the degradation rate was higher and the half-life was shorter.The degradation rate of $\mathrm{pH} 8$ was the fastest, reaching complete degradation at $12 \mathrm{~h}$, and the half-life was the shortest, 5.5h.The second was $\mathrm{pH} 9$, which also reached complete degradation at $12 \mathrm{~h}$ with a half-life of $15.73 \mathrm{~h}$.It can also be seen that $\mathrm{pH} 8$ is the optimal $\mathrm{pH}$ for degradation of biolactam antibiotics by strain PG102, and the optimal degradation range is ph8-9.
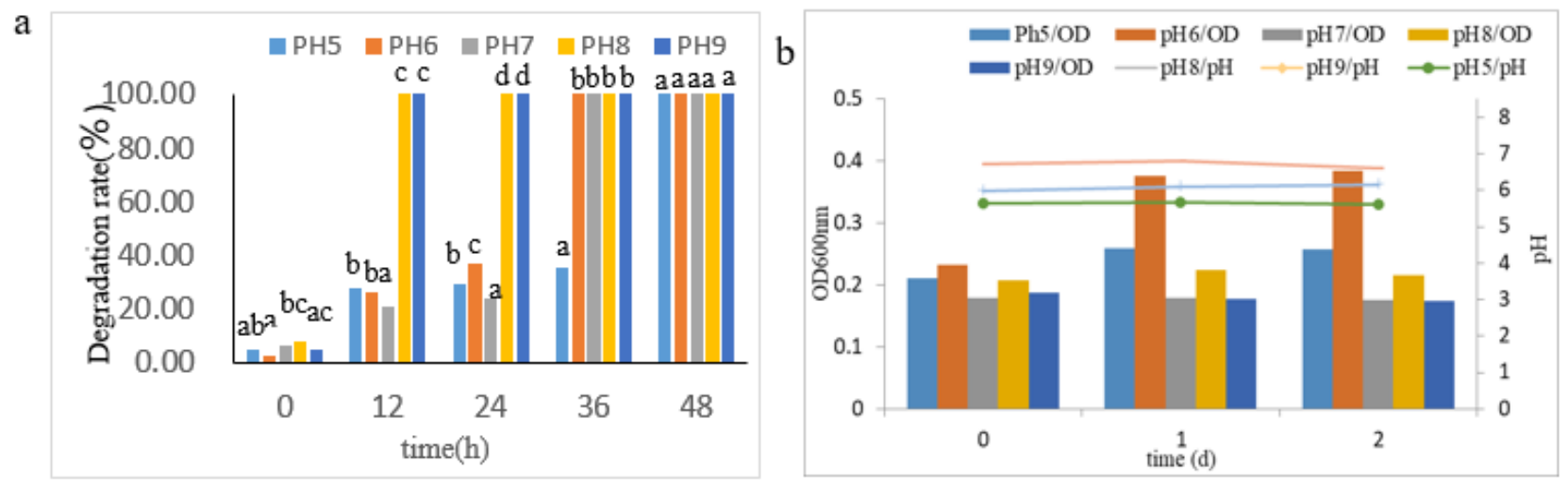

Figure 4. Degradation experiment of strain PG102 at different $\mathrm{pH}$

a: Histogram of degradation rate $\quad$ b:Change of $\mathrm{pH}$ or OD after adding strain PG102

Table2. Kinetic parameters of degradation of strain PG102 under different pH conditions

\begin{tabular}{ccccc}
\hline $\mathrm{pH}$ & Degradation kinetics equation & $\mathrm{C}_{0 / 10}{ }^{-3}$ & $\mathrm{k}$ & $\mathrm{t}_{50} / \mathrm{h}$ \\
\hline 5 & $\mathrm{C}_{\mathrm{t}}=0.05 \mathrm{e}^{-0.023 \mathrm{t}}$ & 0.05 & 0.023 & 30.34 \\
6 & $\mathrm{C}_{\mathrm{t}}=0.05 \mathrm{e}^{-0.031 \mathrm{t}}$ & 0.05 & 0.031 & 22.62 \\
7 & $\mathrm{C}_{\mathrm{t}}=0.05 \mathrm{e}^{-0.029 \mathrm{t}}$ & 0.05 & 0.029 & 23.92 \\
8 & $\mathrm{C}_{\mathrm{t}}=0.05 \mathrm{e}^{-0.126 \mathrm{t}}$ & 0.05 & 0.126 & 5.5 \\
9 & $\mathrm{C}_{\mathrm{t}}=0.05 \mathrm{e}^{-0.044 \mathrm{t}}$ & 0.05 & 0.044 & 15.73 \\
\hline
\end{tabular}

\section{Conclusion}

In this study, PG102 with high degradation efficiency of $\beta$-lactam antibiotics was screened from livestock waste. 16S rRNA gene sequence analysis identified Achromobacter pulmonis. It can degrade 4 kinds of $\beta$-lactam antibiotics, including penicillin potassium, amoxicillin, cefuroxime sodium and ceftiofur sodium. The three antibiotics penicillin potassium, amoxicillin and cefuroxime sodium can be completely degraded in 3 days. The degradation spectrum is broad, the degradation rate is high, and the degradation effect is higher than the data reported in existing literature. Strain PG102 can grow at $55^{\circ} \mathrm{C}$, which provides the basis for the process control of compost biodegradation. Strain PG102 can grow under the conditions of $\mathrm{pH}$ 5-9, and the optimal degradation $\mathrm{pH}$ is 8-9, which also provides a basis for the $\mathrm{pH}$ setting of the strain in practical applications. Therefore, strain PG102 
is a Strains with high efficiency and strong environmental adaptability can efficiently degrade $\beta$-lactam antibiotics under conditions such as high temperature and strong alkali. The results of this study have great application value for antibiotic degradation in the environment. It provides a scientific basis for antibiotic treatment in the environment.

\section{Acknowledgments}

This work was supported by the National Key R \& D Program of China (2018YFD0500206). We also thank the anonymous reviewers for their critical reviews of the manuscript.

\section{References}

[1] L. Monica, Vermillion.Maier, Ronald.S Tjeerdema, Azithromycin sorption and biodegradation in a simulated California river system,Chemosphere.190 (2018) 471-480.

[2] S.T. Lee , H.T. Tran , C. Lin, Characterization of Dioctyl terephthalate biodegradation by food waste composting, Modern Physics Letters B.(2019) 48.

[3] HUA. W, CHU. Y, FANG. C, Occurrence of veterinary antibiotics in swine manure from large-scale feedlots in Zhejiang Province, China, Bulletin of Environmental Contamination \& Toxicology.98 (2017) 472-477.

[4] Amin, Ainatul Mardhiah Mohd, and S. M. Sauid 1, Biodegradation Characteristics of Tacca leontopetaloides Thermoplastic Films under Controlled Composting Conditions, Key Engineering Materials 797(2019) 289-295.

[5] Alexandrino, D.A.M. ,Mucha A. P. , Almeida C. M. R. , Gao W , Jia Z.\& Carvalho M. F, Biodegradation of the veterinary antibiotics enrofloxacin and ceftiofur and associated microbial community dynamics. Science of The Total Environment 581-582(2017) 359-368.

[6] Alexandrino, D. A. M. , Mucha, A. P. , Almeida, C. M. R. , Gao, W., Jia, Z , \& Carvalho, M. F. (2017). Biodegradation of the veterinary antibiotics enrofloxacin and ceftiofur and associated microbial community dynamics. Science of The Total Environment, 581-582, 359-368.

[7] Zhang. H , Dong. M , Yang. Q , Biodegradation of zearalenone by Saccharomyces cerevisiae: Possible involvement of ZEN responsive proteins of the yeast, Journal of Proteomics. (2016) S1874391916301397.

[8] A. Pennacchio, A. Varriale , G. Esposito , A rapid and sensitive assay for the detection of benzylpenicillin (PenG) in milk, Plos One.10 (2015) e0132396.

[9] Li W , Shen H, Hong Y, Zhang Y , \& Zhang, F. Simultaneous determination of 22 cephalosporins drug residues in pork muscle using liquid chromatography-tandem mass spectrometry, Journal of chromatography. B, Analytical technologies in the biomedical and life sciences 1022(2016):298-307.

[10] E. Patyra, K. Kwiatek, Development and validation of multiresidue analysis for tetracycline antibiotics in feed by high performance liquid chromatography coupled to mass spectrometry[J], Food Addit Contam Part A Chem Anal Control Expo Risk Assess, 34 (2017) 1553-1561.

[11] J. Zycka-Krzesinska, J. Boguslawska, T. AleksandrzakPiekarczyk , Identification and characterization of tetracycline resistance in Lactococcus lactis isolated from Polish raw milk and fermented artisanal products[J], Int J Food Microbiol. 211(2015) 134-141. 